\title{
Observing left-right symmetry in the cosmic microwave background
}

\author{
Debasish Borah, ${ }^{1, *}$ Arnab Dasgupta $\odot,{ }^{2, \dagger}$ Chayan Majumdar, ${ }^{3, *}$ and Dibyendu Nanda ${ }^{1, \S}$ \\ ${ }^{1}$ Department of Physics, Indian Institute of Technology Guwahati, Assam 781039, India \\ ${ }^{2}$ Institute of Convergence Fundamental Studies, Seoul-Tech, Seoul 139-743, Korea \\ ${ }^{3}$ Department of Physics, Indian Institute of Technology Bombay, Mumbai 400076, India
}

(Received 9 June 2020; accepted 11 August 2020; published 26 August 2020)

\begin{abstract}
We consider the possibility of probing the left-right symmetric model (LRSM) via the cosmic microwave background (CMB). We adopt the minimal LRSM with Higgs doublets, also known as the doublet leftright model (DLRM), where all fermions including the neutrinos acquire masses only via their couplings to the Higgs bidoublet. Because of the Dirac nature of light neutrinos, there exist additional relativistic degrees of freedom which can thermalize in the early Universe by virtue of their gauge interactions corresponding to the right sector. We constrain the model from Planck 2018 bound on the effective relativistic degrees of freedom and also estimate the prospects for planned CMB stage IV experiments to constrain the model further. We find that the $W_{R}$ boson mass below $4.06 \mathrm{TeV}$ can be ruled out from the Planck 2018 bound at $2 \sigma$ C.L. in the exact left-right symmetric limit which is equally competitive as the LHC bounds from dijet resonance searches. On the other hand, the Planck 2018 bound at $1 \sigma$ C.L. can rule out a much larger parameter space out of reach of present direct search experiments, even in the presence of additional relativistic degrees of freedom around the $\mathrm{TeV}$ corner. We also study the consequence of these constraints on dark matter in the DLRM by considering a right-handed real fermion quintuplet to be the dominant dark matter component in the Universe.
\end{abstract}

DOI: $10.1103 /$ PhysRevD.102.035025

\section{INTRODUCTION}

Left-right symmetric models (LRSMs) [1-12] have been one of the most popular beyond the standard model (BSM) frameworks studied in the literature. Here the gauge symmetry of the SM is extended to $S U(3)_{c} \times S U(2)_{L} \times$ $S U(2)_{R} \times U(1)_{B-L}$ so that the right-handed fermions (which are singlet in the SM) can form doublets under the new $S U(2)_{R}$. This not only makes the inclusion of a right-handed neutrino automatic, but also puts the left- and right-handed fermions on equal footing. Incorporating an additional discrete symmetry (or left-right parity) ensures that the theory is invariant under $S U(2)_{L} \leftrightarrow S U(2)_{R}$. Thus, the model not only explains the origin of parity violation in electroweak interactions through spontaneous breaking of a parity symmetric theory at the high-energy scale but also incorporates a right-handed neutrino, crucial to generate light neutrino masses and mixing observed at neutrino

\footnotetext{
*dborah@iitg.ac.in †arnabdasgupta@protonmail.ch *hayan@phy.iitb.ac.in

§ibyendu.nanda@iitg.ac.in
}

Published by the American Physical Society under the terms of the Creative Commons Attribution 4.0 International license. Further distribution of this work must maintain attribution to the author(s) and the published article's title, journal citation, and DOI. Funded by SCOAP ${ }^{3}$. oscillation experiments $[13,14]$. Apart from the possibility of realizing left-right symmetry as an intermediate symmetry in popular grand unified theories like $S O(10)$, a TeVscale realization of a LRSM can also have very interesting consequences at collider experiments like the Large Hadron Collider (LHC) [15-21].

Conventionally, the very first proposals and studies of LRSMs [1-5] considered a scalar bidoublet for generating fermion masses and also for electroweak symmetry breaking, whereas a pair of scalar doublets were introduced for the purpose of left-right symmetry breaking at the highenergy scale. A very recent detailed study of this model can be found in Ref. [22]. On the other hand, the LRSM proposals put forward later [7-12] received much more attention due to the possibility of a seesaw origin of light neutrino masses through a combination of a type-I seesaw [23-26] and a type-II seesaw [8,27-30] or a type-III seesaw [31]. In the doublet left-right model (DLRM), in its minimal version, there is no such seesaw mechanism, as all fermions including neutrinos acquire Dirac masses by virtue of their couplings to the bidoublet scalar. While generating a sub-eV neutrino mass in this fashion requires relevant Yukawa couplings at the level of $<10^{-12}$, we adopt this minimal scenario to study some of the interesting phenomenological consequences. Radiative generation of light Dirac neutrinos in different left-right symmetric models has also been discussed over the past few decades 
[32-39] which may provide a UV completion of the minimal DLRM we discuss here. Since such UV completions do not drastically change the conclusions we reach in the present work, we stick to the DLRM for the sake of simplicity.

The Dirac nature of light neutrinos in the DLRM gives rise to additional relativistic degrees of freedom which can be thermalized in the early Universe due to their gauge interactions mediated by right sector gauge bosons. Such additional light degrees of freedom can be probed by precise measurements of the cosmic microwave background (CMB) anisotropies. Recent 2018 data from the CMB measurement by the Planck satellite [40] suggests the effective degrees of freedom for neutrinos as

$$
N_{\text {eff }}=2.99_{-0.33}^{+0.34}
$$

at $2 \sigma$ or $95 \%$ C.L. including baryon acoustic oscillation data. At $1 \sigma$ C.L., it becomes more stringent to $N_{\text {eff }}=2.99 \pm 0.17$. Both these bounds are consistent with the SM prediction $N_{\mathrm{eff}}^{\mathrm{SM}}=3.045$ [41-43]. Upcoming CMB stage IV (CMB-S4) experiments are expected to put much more stringent bounds than Planck due to their potential of probing all the way down to $\Delta N_{\text {eff }}=N_{\text {eff }}-N_{\text {eff }}^{\mathrm{SM}}=0.06$ [44]. We use the existing constraints and put strong limits on the scale of left-right symmetry or, equivalently, the right sector gauge bosons $W_{R}$ and $Z_{R}$. For comparison, we also check the corresponding bounds for the left-right asymmetric scenario by considering different $S U(2)_{R}$ gauge couplings. Interestingly, we find that the bounds on $W_{R}$ and $Z_{R}$ mass from the Planck 2018 bound on $\Delta N_{\text {eff }}$ at $2 \sigma$ C.L. are equally competitive as the latest LHC bounds $[16,17,20]$ and much stronger than the corresponding bounds from flavor physics [45]. On the other hand, the same Planck 2018 bound at $1 \sigma$ C.L. can rule out a much larger mass window for $W_{R}$ and $Z_{R}$ out of reach of present collider experiments. In fact, CMB-S4 will be able to probe a much larger region of $W_{R}$ and $Z_{R}$ masses out of existing collider reach and, hence, can probe or rule out the minimal model. Since there have been a few recent studies on the gauged $B-L$ model with light Dirac neutrinos [46-49] and corresponding constraints due to the Planck 2018 bound on $\Delta N_{\text {eff }}$, we also reproduce the corresponding parameter space in the gauged $B-L$ model and compare with the one obtained in the DLRM. We point out the important difference due to the restricted range of DLRM gauge couplings $g_{R}$ and $g_{B L}$ unlike that in the gauged $B-L$ model. We also show the impact of these constraints on dark matter (DM) parameter space in the DLRM by considering a right-handed fermion quintuplet to be the dominant component of DM which can thermalize by virtue of its interactions with the SM mediated by right sector gauge bosons. We calculate the parameter space allowed from the observed DM relic and find the leftover parameter space after applying the $\Delta N_{\text {eff }}$ bound. Finally, we comment on the more stringent Planck $20181 \sigma$ bound which can be satisfied if more light fields below the scale of left-right symmetry breaking in addition to the SM plus three right-handed neutrinos exist. In fact, we show that the DLRM with right-handed fermion quintuplet DM can give rise to a sufficient number of additional degrees of freedom to serve this purpose.

This paper is organized as follows. In Sec. II, we discuss the doublet left-right symmetric model followed by a discussion of additional relativistic degrees of freedom due to light Dirac neutrinos in Sec. III. In Sec. IV, we briefly discuss dark matter in the DLRM, particularly focusing on fermion quintuplet DM, followed by results and discussion in Sec. V. We finally conclude in Sec. VI.

\section{THE DLRM}

We briefly discuss the doublet left-right symmetric model in this section. The fermion and scalar content of the model are given in Tables I and II, respectively. The relevant Yukawa Lagrangian giving masses to the three generations of leptons is given by

$$
\mathcal{L}=h_{i j} \bar{l}_{L, i} \Phi l_{R, j}+{\widetilde{h_{i j}}}_{L, i} \tilde{\Phi} l_{R, j}+\text { H.c. },
$$

where the indices $i, j=1,2,3$ represent the family indices for the three generations of fermions, $\widetilde{\Phi}=\tau_{2} \phi^{*} \tau_{2}$, and $\tau_{2}$ is the Pauli matrix. The gauge structure of the model prevents any renormalizable Yukawa couplings involving the scalar doublets $\chi_{L, R}$. The scalar potential $V_{\text {scalar }}$ is given by [22]

$$
\begin{aligned}
V_{\text {scalar }}= & -\mu_{1}^{2} \operatorname{Tr}\left[\Phi^{\dagger} \Phi\right]-\mu_{2}^{2} \operatorname{Tr}\left[\Phi^{\dagger} \tilde{\Phi}+\tilde{\Phi}^{\dagger} \Phi\right]-\mu_{3}^{2}\left(\chi_{L}^{\dagger} \chi_{L}+\chi_{R}^{\dagger} \chi_{R}\right)+\lambda_{1}\left(\operatorname{Tr}\left[\Phi^{\dagger} \Phi\right]\right)^{2}+\lambda_{2}\left\{\left(\operatorname{Tr}\left[\Phi^{\dagger} \tilde{\Phi}\right]\right)^{2}+\left(\operatorname{Tr}\left[\tilde{\Phi}^{\dagger} \Phi\right]\right)^{2}\right\} \\
& +\lambda_{3} \operatorname{Tr}\left[\Phi^{\dagger} \tilde{\Phi}\right] \operatorname{Tr}\left[\tilde{\Phi}^{\dagger} \Phi\right]+\lambda_{4} \operatorname{Tr}\left[\Phi^{\dagger} \Phi\right] \operatorname{Tr}\left[\Phi^{\dagger} \tilde{\Phi}+\tilde{\Phi}^{\dagger} \Phi\right]+\mu_{1}^{\prime}\left(\chi_{L}^{\dagger} \Phi \chi_{R}+\chi_{R}^{\dagger} \Phi^{\dagger} \chi_{L}\right)+\mu_{2}^{\prime}\left(\chi_{L}^{\dagger} \tilde{\Phi} \chi_{R}+\chi_{R}^{\dagger} \tilde{\Phi}^{\dagger} \chi_{L}\right) \\
& +\rho_{1}\left[\left(\chi_{L}^{\dagger} \chi_{L}\right)^{2}+\left(\chi_{R}^{\dagger} \chi_{R}\right)^{2}\right]+\alpha_{1} \operatorname{Tr}\left[\Phi^{\dagger} \Phi\right]\left[\left(\chi_{L}^{\dagger} \chi_{L}\right)+\left(\chi_{R}^{\dagger} \chi_{R}\right)\right]+\alpha_{2} e^{i \delta}\left[\operatorname{Tr}\left[\tilde{\Phi} \Phi^{\dagger}\right]\left(\chi_{L}^{\dagger} \chi_{L}\right)+\operatorname{Tr}\left[\Phi \tilde{\Phi}^{\dagger}\right]\left(\chi_{R}^{\dagger} \chi_{R}\right)\right] \\
& +\alpha_{2} e^{-i \delta}\left[\operatorname{Tr}\left[\tilde{\Phi}^{\dagger} \Phi\right]\left(\chi_{L}^{\dagger} \chi_{L}\right)+\operatorname{Tr}\left[\Phi^{\dagger} \tilde{\Phi}\right]\left(\chi_{R}^{\dagger} \chi_{R}\right)\right]+\alpha_{3}\left(\chi_{L}^{\dagger} \Phi \Phi^{\dagger} \chi_{L}+\chi_{R}^{\dagger} \Phi^{\dagger} \Phi \chi_{R}\right)+\alpha_{4}\left(\chi_{L}^{\dagger} \tilde{\Phi}^{\dagger} \chi_{L}+\chi_{R}^{\dagger} \tilde{\Phi}^{\dagger} \tilde{\Phi} \chi_{R}\right) .
\end{aligned}
$$

For details of the minimization of the scalar potential and resulting symmetry breaking, please refer to Ref. [22]. In the symmetry-breaking pattern, the neutral component of the Higgs doublet $\chi_{R}$ acquires a vacuum expectation value (VEV) to break the gauge symmetry of the DLRM into that of the SM and then to the $U(1)$ of electromagnetism by the VEV of the neutral components of Higgs bidoublet $\Phi$ : 
TABLE I. Fermionic fields of the present model including the SM fermions.

\begin{tabular}{lc}
\hline \hline Particles & $S U(3)_{c} \times S U(2)_{L} \times S U(2)_{R} \times U(1)_{B-L}$ \\
\hline$Q_{L}=\left(\begin{array}{l}u_{L} \\
d_{L}\end{array}\right)$ & $\left(3,2,1, \frac{1}{3}\right)$ \\
$Q_{R}=\left(\begin{array}{l}u_{R} \\
d_{R}\end{array}\right)$ & $\left(3,1,2, \frac{1}{3}\right)$ \\
$\ell_{L}=\left(\begin{array}{l}\nu_{L} \\
e_{L}\end{array}\right)$ & $(1,2,1,-1)$ \\
$\ell_{R}=\left(\begin{array}{l}\nu_{R} \\
e_{R}\end{array}\right)$ & $(1,1,2,-1)$ \\
\hline \hline
\end{tabular}

TABLE II. Scalar fields and their corresponding charges under all the symmetry groups.

\begin{tabular}{lc}
\hline \hline Particles & $S U(3)_{c} \times S U(2)_{L} \times S U(2)_{R} \times U(1)_{B-L}$ \\
\hline$\Phi=\left(\begin{array}{cc}\phi^{0} \phi_{\phi^{\prime}+} \\
\phi^{0}\end{array}\right)$ & $(1,2,2,0)$ \\
$\chi_{L}$ & $(1,2,1,1)$ \\
$\chi_{R}$ & $(1,1,2,1)$ \\
\hline \hline
\end{tabular}

$$
S U(2)_{L} \times S U(2)_{R} \times U(1)_{B-L}\langle\underline{\langle}\rangle S U(2)_{L} \times U(1)_{Y}
$$

$$
\stackrel{\langle\Phi\rangle}{\longrightarrow} U(1)_{\mathrm{em}} \text {. }
$$

The VEVs of the neutral components of the Higgs fields can be denoted as

$$
\langle\Phi\rangle=\left(\begin{array}{cc}
\frac{k_{1}}{\sqrt{2}} & 0 \\
0 & \frac{k_{2}}{\sqrt{2}}
\end{array}\right), \quad\left\langle\chi_{L}\right\rangle=\left(\begin{array}{c}
0 \\
\frac{v_{L}}{\sqrt{2}}
\end{array}\right), \quad\left\langle\chi_{R}\right\rangle=\left(\begin{array}{c}
0 \\
\frac{v_{R}}{\sqrt{2}}
\end{array}\right),
$$

where the VEV's $k_{1}$ and $k_{2}$ satisfy the VEV of the SM; namely, $v_{\mathrm{SM}}=\sqrt{k_{1}^{2}+k_{2}^{2}} \approx 246 \mathrm{GeV}$. The spontaneous breaking of DLRM gauge symmetry down to $U(1)_{\mathrm{em}}$ results in two charged massive vector bosons $W_{L}$ and $W_{R}$, two neutral massive bosons $Z_{L}$ and $Z_{R}$, and a massless photon as expected. The details of the mass spectrum of gauge bosons are shown in Appendix A.

The light Dirac neutrino mass and charged lepton mass are given, respectively, by

$$
M_{\nu}=\frac{1}{\sqrt{2}}\left(k_{1} h+k_{2} \widetilde{h}\right), \quad M_{l}=\frac{1}{\sqrt{2}}\left(k_{2} h+k_{1} \widetilde{h}\right),
$$

where the family indices are suppressed. Without any loss of generality, we make use of rotation in the $S U(2)_{L} \times$ $S U(2)_{R}$ space so that only one of the neutral components of the Higgs bidoublet acquires a large vacuum expectation value, $k_{1} \approx v_{\mathrm{SM}}$ and $k_{2} \approx 0$. Under these assumptions, the Dirac neutrino mass matrix is

$$
M_{\nu}=\frac{1}{\sqrt{2}}\left(k_{1} h\right)
$$

while the charged lepton mass matrix is

$$
M_{l}=\frac{1}{\sqrt{2}}\left(k_{1} \widetilde{h}\right)
$$

Therefore, tiny sub-eV Dirac neutrino mass arises due to the smallness of Yukawa coupling $h$, while charged lepton masses are generated by corresponding Yukawa coupling $\widetilde{h}$. The details of fermion-gauge boson couplings are shown in Appendix B. The details of the scalar mass spectrum are not derived here, as we do not need them for our analysis, and we refer to Ref. [22] for details of the same.

\section{III. $\Delta N_{\text {eff }}$ IN DLRM}

The effective number of relativistic degrees of freedom is defined as

$$
N_{\mathrm{eff}} \equiv \frac{8}{7}\left(\frac{11}{4}\right)^{4 / 3}\left(\frac{\rho_{\mathrm{rad}}-\rho_{\gamma}}{\rho_{\gamma}}\right)
$$

where $\rho_{\text {rad }}=\rho_{\gamma}+\rho_{\nu}$ is the net radiation content of the Universe. As mentioned earlier, the SM prediction is $N_{\mathrm{eff}}^{\mathrm{SM}}=$ 3.045 [41-43], which is also consistent with the constraint from precision measurement of $Z$ boson decay width at large electron positron (LEP) collider $N_{\nu}=2.984 \pm 0.008$ [14]. Any deviation of $N_{\text {eff }}$ from $N_{\text {eff }}^{\mathrm{SM}}$ will therefore indicate the presence of additional relativistic species thermalized in the early Universe. While these additional relativistic degrees of freedom cannot fully thermalize with the SM bath through interactions mediated by a $Z$ boson due to the strong LEP bound, they can thermalize via additional interactions or mediating particles not yet observed in direct search experiments. The right-handed neutrinos in the DLRM provide such an example. They can thermalize with the SMbath in the early Universe due to the interactions mediated by right sector gauge bosons, as depicted by the Feynman diagrams shown in Fig. 1. We consider negligible mixing between left and right sector gauge bosons and, hence, ignore the contributions coming from processes like $\overline{\nu_{R}} \nu_{L} \rightarrow f \bar{f}$ and $\overline{\nu_{L}} \nu_{R} \rightarrow f \bar{f}$. Additionally, the scalar-mediated interactions are negligible due to tiny Dirac Yukawa couplings.

To estimate the contribution in $\Delta N_{\text {eff }}$, we need to check the decoupling temperature of the right-handed neutrinos. The decoupling occurs when the expansion rate of the Universe becomes more than the interaction rate. Hence, the decoupling temperature can be calculated from the following equality:

$$
\Gamma\left(T_{\nu_{R}}^{\mathrm{d}}\right)=H\left(T_{\nu_{R}}^{\mathrm{d}}\right)
$$
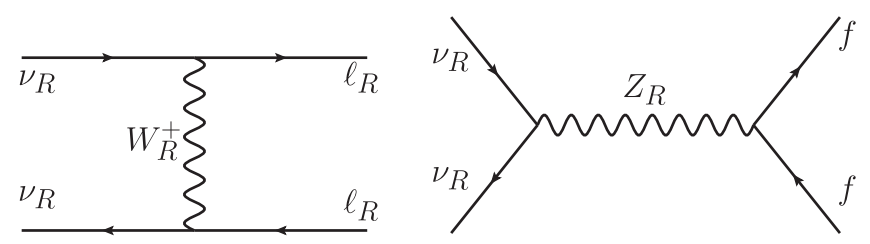

FIG. 1. Feynman diagrams of $\nu_{R}$ annihilation. Here, $l_{R} \equiv$ $e_{R}, \mu_{R}, \tau_{R}$ and $f \equiv e_{R}, \mu_{R}, \tau_{R}, q_{R}$ with $q$ being a quark. 
where $\Gamma(T)$ is the interaction rate and $H(T)$ is the expansion rate of the Universe. The interaction rate can be written as

$$
\Gamma(T)=n_{\nu_{R}}(T)\left\langle\sigma_{\mathrm{Tot}} v\right\rangle,
$$

where the number density $n_{\nu_{R}}$ for a relativistic neutrino can be written as

$$
n_{\nu_{R}}(T)=\frac{3 g_{\nu_{R}}}{4 \pi^{2}} \zeta(3) T^{3}
$$

and the annihilation cross sections of right-handed neutrinos are given in Appendix C.

The expansion rate of the Universe can be written as

$$
\begin{aligned}
H(T) & =\sqrt{\frac{8 \pi G_{N} \rho(T)}{3}} \\
& =\sqrt{\frac{4 \pi^{3} G_{N}}{45}\left(g_{*}(T)+3 \frac{7}{8} g_{\nu_{R}}\right)} T^{2},
\end{aligned}
$$

where $g_{\nu_{R}}$ is the internal degrees of freedom for righthanded neutrinos. Thus, the contribution of $\nu_{R}$ to effective relativistic degrees of freedom can be estimated as

$\Delta N_{\mathrm{eff}}=N_{\mathrm{eff}}-N_{\mathrm{eff}}^{\mathrm{SM}}=N_{\nu_{R}}\left(\frac{T_{\nu_{R}}}{T_{\nu_{L}}}\right)^{4}=N_{\nu_{R}}\left(\frac{g_{* s}\left(T_{\nu_{L}}^{\mathrm{d}}\right)}{g_{* s}\left(T_{\nu_{R}}^{\mathrm{d}}\right)}\right)^{4 / 3}$,

where $N_{\nu_{R}}$ represents the number of relativistic righthanded neutrinos, $g_{*}(T)$ corresponds to the relativistic degrees of freedom at temperature $T, g_{* s}(T)$ corresponds to the relativistic entropy degrees of freedom at temperature $T,{ }^{1}$ and $T_{\nu_{R}}^{\mathrm{d}}$ and $T_{\nu_{L}}^{\mathrm{d}}$ are the decoupling temperatures for $\nu_{R}$ and $\nu_{L}$, respectively. Thus, depending upon the decoupling temperature of $\nu_{R}$ and, hence, $g_{*}\left(T_{\nu_{R}}^{\mathrm{d}}\right)$, the additional contribution to $\Delta N_{\text {eff }}$ can be kept within experimental upper limits. The lower the strength of $\nu_{R}$ interaction with the SM bath or the higher the mediator mass of $\nu_{R}$-SM interactions, the larger will be $g_{*}\left(T_{\nu_{R}}^{\mathrm{d}}\right)$ and, hence, the smaller will be $\Delta N_{\text {eff }}$. A similar analysis for $U(1)_{B-L}$ extension of the SM can be found in Refs. [46-49], whereas some estimates in the context of radiative Dirac neutrino mass in LRSM were made in Refs. [35,37].

\section{DARK MATTER IN DLRM}

The data from the Planck experiment which restrict the effective relativistic degrees of freedom in our Universe also reveal that more than $26 \%$ of the present Universe's energy density is composed of a nonluminous and nonbaryonic form of matter, known as dark matter. Apart from recent cosmology-based experiments like Planck, there have been

\footnotetext{
${ }^{1}$ We use $g_{*}$ and $g_{* s}$ interchangeably, which is true in the SM at high temperatures.
}

several astrophysical evidences for many decades suggesting the presence of DM [50-52]. In terms of density parameter $\Omega_{\mathrm{DM}}$ and $h=$ Hubble parameter $/\left(100 \mathrm{~km} \mathrm{~s}^{-1} \mathrm{Mpc}^{-1}\right)$, the present DM abundance is conventionally reported as [40] $\Omega_{\mathrm{DM}} h^{2}=0.120 \pm 0.001$ at $68 \%$ C.L. Given that none of the SM particles can be a viable DM candidate, several BSM proposals have been put forward, among which the weakly interacting massive particle (WIMP) paradigm is the most popular one. In this framework, a DM particle having masses and interactions similar to those around the electroweak scale gives rise to the observed relic after thermal freeze-out, a remarkable coincidence often referred to as the WIMP miracle [53].

The minimal DLRM discussed above does not have a stable DM candidate. One can, however, minimally extend the model by including additional scalar or fermionic multiplets in the spirit of the minimal dark matter scenario [54-56]. In these models, the dark matter candidate is stabilized either by a $Z_{2}=(-1)^{B-L}$ subgroup of the $U(1)_{B-L}$ gauge symmetry or due to an accidental symmetry at the renormalizable level due to the absence of any renormalizable operator leading to dark matter decay. Such a minimal dark matter scenario in the LRSM has been studied recently by the authors of Refs. [57,58]. Some more recent works on DM in the LRSM can be found in Refs. [35-37,59-66]. Unlike in the triplet LRSM, where $S U(2)_{R} \times U(1)_{B-L}$ gauge symmetry is spontaneously broken by a scalar triplet with even $(B-L)$ charge $( \pm 2)$, in the DLRM the same happens due to a scalar doublet with odd $(B-L)$ charge $( \pm 1)$. Thus, there is no stabilizing symmetry like $Z_{2}=(-1)^{B-L}$ in the DLRM to stabilize DM. Therefore, some DM candidates like the fermion triplet, fermion bidoublet, and scalar doublet discussed in the context of the triplet LRSM $[57,58]$ are no longer stable in the DLRM due to the presence of renormalizable interactions with lighter fields. We therefore consider the option of a larger fermion multiplet as DM, and the minimal scenario is to consider a real fermion quintuplet of $B-L$ charge 0 . Since we want to constrain the right sector gauge bosons from the cosmology bound on $\Delta N_{\text {eff }}$, we particularly focus on right-handed fermion quintuplet DM whose relic abundance depends upon the strength of its annihilation through right sector gauge bosons.

In the pure left-right symmetric setup, one has to introduce a pair of left- and right-handed fermion quintuplets (having the same mass) which can be written in component form as

$$
\Omega_{L}=\left(\begin{array}{c}
\Omega_{L}^{++} \\
\Omega_{L}^{+} \\
\Omega_{L}^{0} \\
\Omega_{L}^{-} \\
\Omega_{L}^{--}
\end{array}\right), \quad \Omega_{R}=\left(\begin{array}{c}
\Omega_{R}^{++} \\
\Omega_{R}^{+} \\
\Omega_{R}^{0} \\
\Omega_{R}^{-} \\
\Omega_{R}^{--}
\end{array}\right)
$$


Since we are discussing a general scenario with $g_{L} \neq g_{R}$, we consider the left fermion quintuplet to be very heavy and decoupled from the low-energy phenomenology. Even in the pure left-right symmetric limit $g_{L}=g_{R}$, one can make the left quintuplet decouple from the low-energy phenomenology by introducing a parity-odd scalar singlet whose nonzero VEV at a very high scale splits the right and left fermion masses. Such proposals where the left-right discrete symmetry or parity gets broken spontaneously before $S U(2)_{R} \times U(1)_{B-L}$ gauge symmetry were put forward long ago in Refs. [67-69]. While all the components of the fermion multiplet have the same tree-level masses, at the radiative level, there arises a mass splitting between charged (with $Q$ ) and neutral components given by [57,58]

$$
\begin{aligned}
M_{\Omega_{R}^{Q}}-M_{\Omega_{R}^{0}} \simeq & \frac{\alpha_{2}}{4 \pi} \frac{g_{R}^{2}}{g_{L}^{2}} M Q^{2}\left[f\left(r_{W_{R}}\right)-c_{M}^{2} f\left(r_{Z_{R}}\right)\right. \\
& \left.-s_{W}^{2} s_{M}^{2} f\left(r_{Z_{L}}\right)-c_{W}^{2} s_{M}^{2} f\left(r_{\gamma}\right)\right],
\end{aligned}
$$

where $s_{M}=\sin \theta_{M} \equiv \tan \theta_{W} \frac{g_{L}}{g_{R}}, s_{W}=\sin \theta_{W}, r_{X}=M_{X} / M$, and

$$
f(r) \equiv 2 \int_{0}^{1} d x(1+x) \log \left[x^{2}+(1-x) x^{2}\right] .
$$

The bare mass of the multiplet is denoted by $M$. Here, the one-loop self-energy corrections through mediations of gauge bosons are presented within the square bracket of the second expression. Because of such a tiny one-loop mass splitting, the next-to-lightest component of each DM multiplet can be thermally accessible during the dark matter freeze-out, and, hence, the coannihilation effects play a crucial role [70]. While in the triplet LRSM there exists the possibility that, for $M \gg M_{W_{R}}$, the neutral component of the multiplet can become heavier compared to the charged components, such possibilities do not arise in the DLRM. This was noted in Ref. [66], which we also confirm. Apart from its role in enhancing DM coannihilations, such a mass splitting, if small enough, may also induce inelastic DMnucleon scattering mediated by $W_{R}$ bosons. However, for our region of interest, such a tiny mass splitting does not arise. This ensures that $W_{R}$-mediated DM-nucleon scattering occurs only at one-loop level and, hence, remains suppressed. Spin-independent elastic DM nucleon scattering mediated by $Z_{R}$ at tree level remains absent due to vanishing $B-L$ charge of the fermion quintuplet discussed in this work. Thus, the DM phenomenology of a right-handed fermion quintuplet is mainly governed by its gauge interactions, which are given by [66]

$$
\begin{aligned}
\mathcal{L}_{\Omega_{R}} \supset & -s_{W} s_{M} g_{R} Q \overline{\Omega_{R}^{Q}} Z_{L}^{\mu} \gamma_{\mu} \Omega_{R}^{Q}+c_{M} g_{R} Q \overline{\Omega_{R}^{Q}} Z_{R}^{\mu} \gamma_{\mu} \Omega_{R}^{Q} \\
& +c_{W} s_{M} g_{R} Q \overline{\Omega_{R}^{Q}} A^{\mu} \gamma_{\mu} \Omega_{R}^{Q} \\
& +\frac{g_{R}}{\sqrt{2}}\left(c_{Q} \overline{\Omega_{R}^{Q+1}} W_{R}^{\mu} \gamma_{\mu} \Omega_{R}^{Q}+\text { H.c. }\right),
\end{aligned}
$$

where $c_{Q}=\sqrt{(2+Q+1)(2-Q)}$ and $Q$ is the electromagnetic charge of the quintuplet component.

\section{RESULTS AND DISCUSSION}

Using the recipe discussed in the previous section, we first calculate the decoupling temperature of right-handed neutrinos from the thermal bath for different values of $W_{R}$ and $Z_{R}$ mass and gauge coupling $g_{R}$. The variation of decoupling temperature with $W_{R}$ mass for different values of $g_{R}$ is shown in Fig. 2. Although both $W_{R}$ and $Z_{R}$ masses play a role in right-handed neutrino interactions with the thermal bath, we show the variation of the decoupling temperature as well as other physical quantities only in terms of $W_{R}$ mass. This is due to the fact that $Z_{R}$ mass typically depends upon $W_{R}$ mass and is heavier than it, similar to $Z$ and $W$ masses of the SM. Also, we are not restricting ourselves to pure left-right symmetric limit $g_{R}=g_{L}$ and considering different values of $g_{R}$ as well. The decoupling temperature rises for lower values of gauge coupling as well as higher values of $W_{R}$ mass as seen from Fig. 2, which is expected as the corresponding rate of interactions decreases. Typically, the interaction cross section $\langle\sigma v\rangle$ of right-handed neutrinos depends upon the temperature as $g_{R}^{4} T^{2} / M_{W_{R}}^{4}$, and, hence, the rate of interaction is $\Gamma(T) \propto g_{R}^{4} T^{5} / M_{W_{R}}^{4}$. When this is compared with the Hubble parameter $H(T) \propto T^{2}$ as in Eq. (7) to find the decoupling temperature $T_{\nu_{R}}^{\mathrm{d}}$, it is clear that, with an increase in $W_{R}$ mass, the decoupling temperature will also rise. Similarly, a decrease in $g_{R}$ will lead to an increase in the decoupling temperature for same value of $M_{W_{R}}$. This

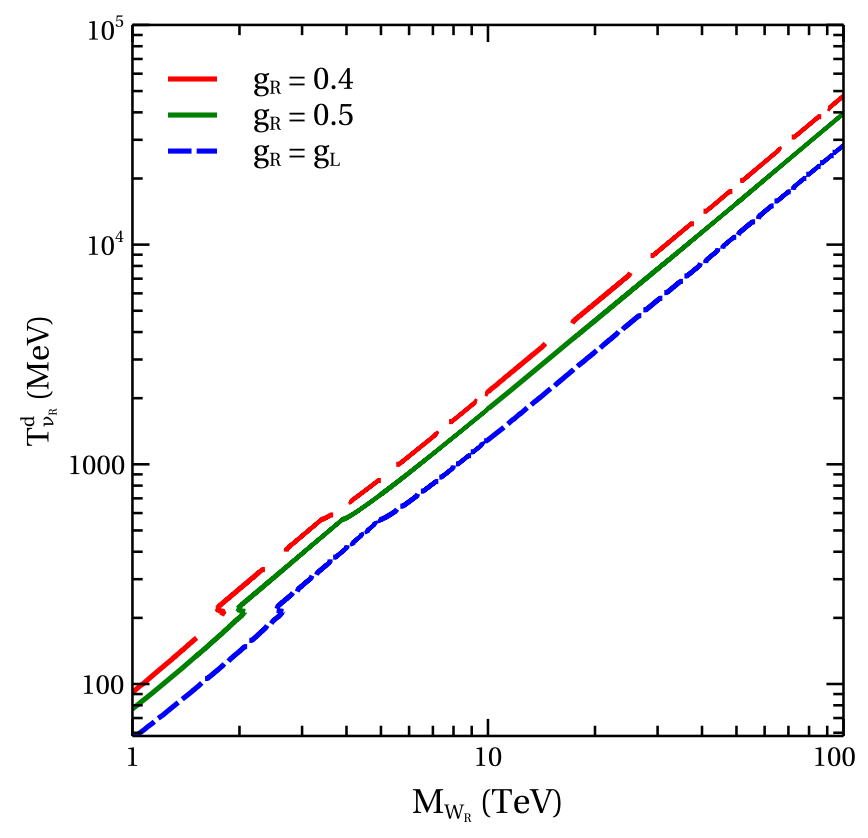

FIG. 2. Decoupling temperature of right-handed neutrinos as a function of the $W_{R}$ mass for different gauge couplings $g_{R}$. 

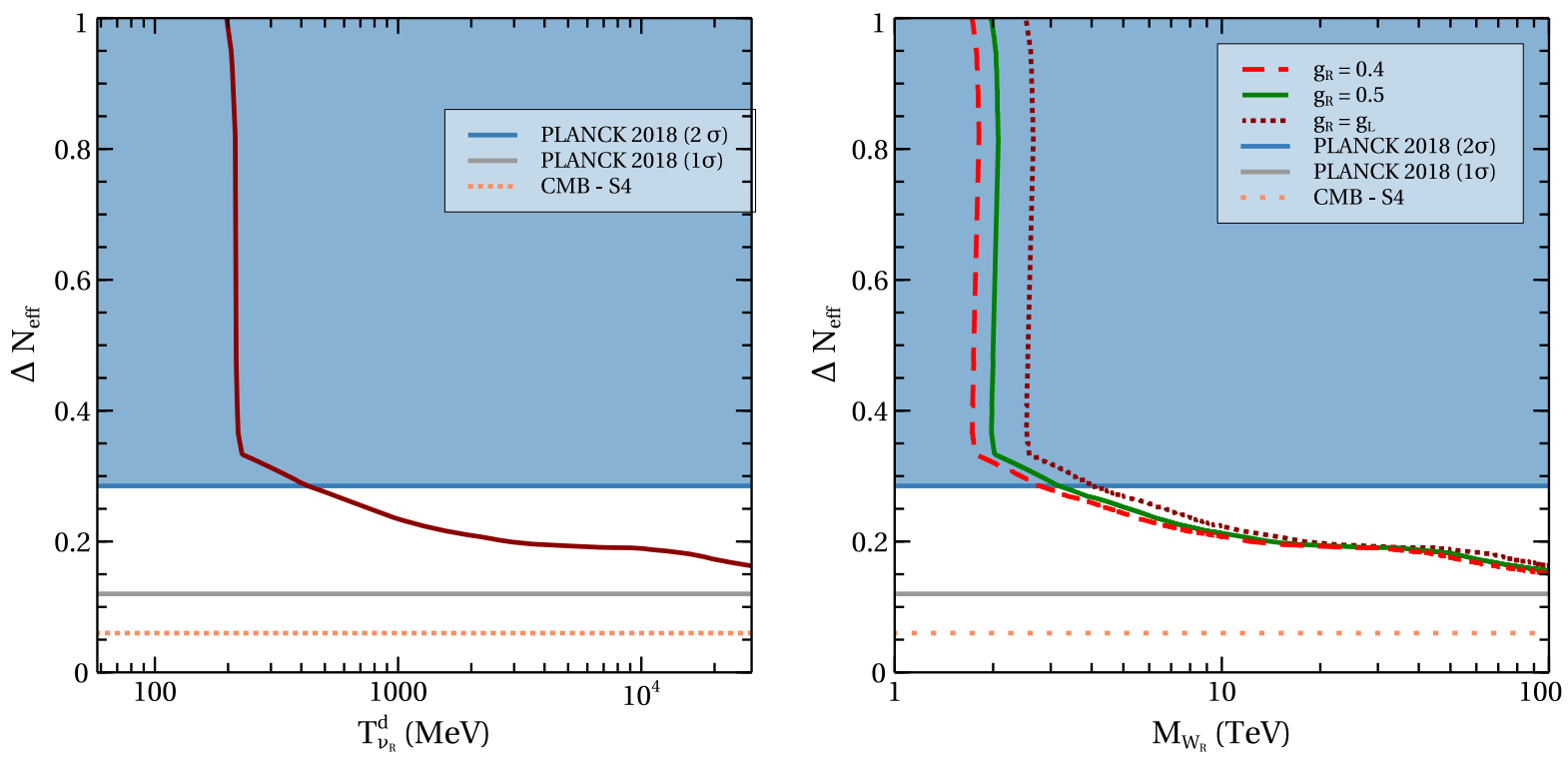

FIG. 3. $\Delta N_{\text {eff }}$ as a function of the decoupling temperature (left panel) and $W_{R}$ mass (right panel).

conclusion reached from approximate analytical formulas for the cross section and Hubble parameter also agrees with our numerical results shown in Fig. 2.

We then show the contribution to $\Delta N_{\text {eff }}$ in Fig. 3 as functions of the decoupling temperature as well as the $W_{R}$ mass. The dependence of $\Delta N_{\text {eff }}$ on $T_{\nu_{R}}^{\mathrm{d}}$ in the left panel in Fig. 3 can be understood from Eq. (11). The total number of relativistic degrees of freedom at decoupling temperature $g_{* s}\left(T_{\nu_{R}}^{\mathrm{d}}\right)$ increases with the increasing $T_{\nu}^{\mathrm{d}}$, which thereby decreases the value of $\Delta N_{\text {eff }}$. However, at some point $g_{* s}$, it will reach its maximum value and $\Delta N_{\text {eff }}$ becomes almost constant, as seen from the plateau region on the bottom left corner of the left panel in Fig. 3. Since $T_{\nu_{R}}^{\mathrm{d}}$ is being varied independently and the corresponding $g_{* s}\left(T_{\nu_{R}}^{\mathrm{d}}\right)$ is used to find $\Delta N_{\text {eff }}$ using Eq. (11), the behavior of this plot does not depend upon $g_{R}$ and $M_{W_{R}}$. To show the dependence of $\Delta N_{\text {eff }}$ on such model parameters, we have made the plot shown in right panel in Fig. 3. The right panel in Fig. 3 represents the dependence of $\Delta N_{\text {eff }}$ on $M_{W_{R}}$ for three benchmark values of $g_{R}$. One can see that the contribution to the $\Delta N_{\text {eff }}$ decreases with increasing $M_{W_{R}}$. This is because, for higher values of $M_{W_{R}}, \nu_{R}$ will decouple at some higher temperature and the contribution to the $\Delta N_{\text {eff }}$ will become smaller. Along with the Planck 2018 bound mentioned earlier, we also show the CMB-S4 sensitivity [71] as well as the Planck $20181 \sigma$ limit, while the latter is the same as SPT-3G sensitivity [72]. Clearly, the Planck 2018 bound at $2 \sigma$ C.L. itself rules out a $W_{R}$ mass below $4.06 \mathrm{TeV}$ with gauge coupling $g_{R}=g_{L}$. On the other hand, a future probe will be able to either confirm or rule out the model, even for very high $W_{R}$ masses, out of reach of direct search experiments. Finally, we show the final parameter space in the $g_{R}-M_{W_{R}}$ plane after applying Planck $20182 \sigma$ constraints in Fig. 4.
Unlike in Refs. [46-49], where similar constraints on the $U(1)_{B-L}$ gauge boson were obtained, the crucial difference in the DLRM is that here one cannot tune the gauge couplings for a particular value of gauge boson mass in order to suppress the contribution to $\Delta N_{\text {eff }}$. This is because the gauge couplings of $S U(2)_{R}$ and $U(1)_{B-L}$ are not arbitrary but related to the gauge coupling of $U(1)_{Y}$ (at the scale of left-right symmetry breaking) as

$$
\frac{1}{g_{Y}^{2}}=\frac{1}{g_{R}^{2}}+\frac{1}{g_{B L}^{2}}
$$

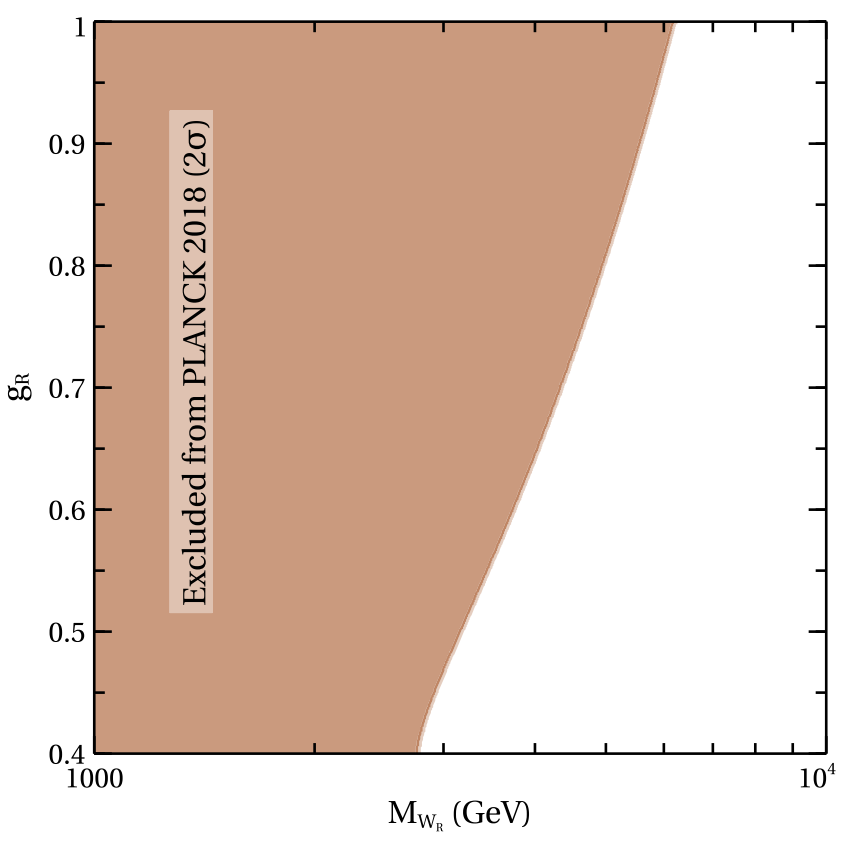

FIG. 4. Parameter space in the $g_{R}-M_{W_{R}}$ plane from Planck 2018 $2 \sigma$ constraints on $\Delta N_{\text {eff }}$. 


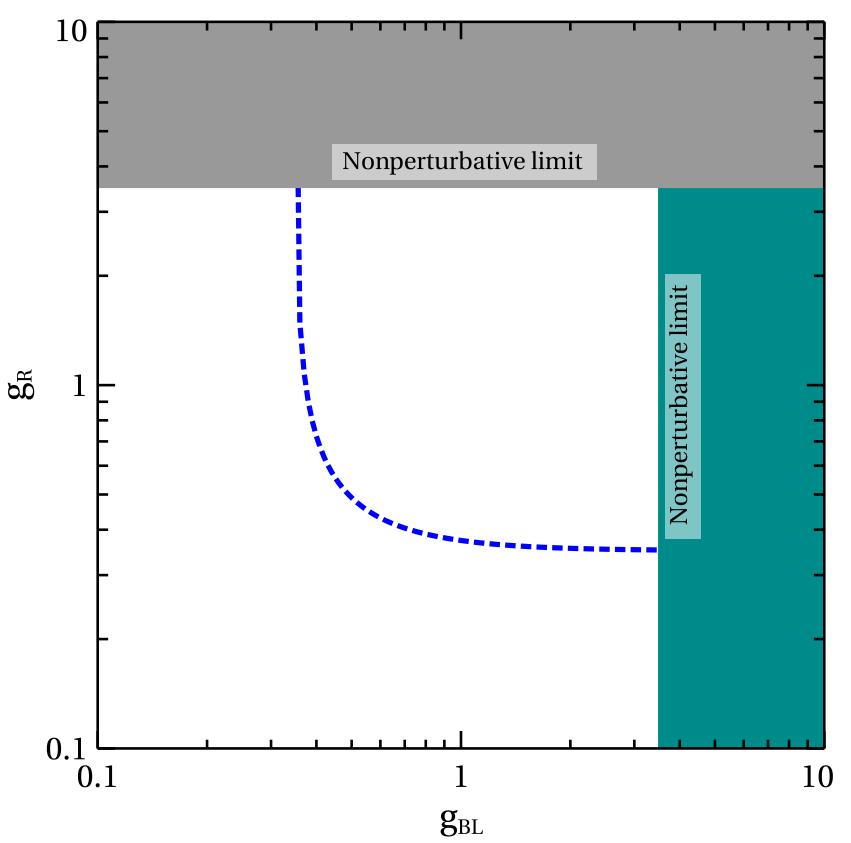

FIG. 5. Allowed values of $g_{B L}$ and $g_{R}$ which will reproduce the value $g_{Y}$ after the spontaneous breaking of $S U(2)_{R} \times U(1)_{B-L}$ to the remaining $U(1)_{Y}$.

Since $g_{Y}$ is known, one cannot change $g_{R}$ and $g_{B L}$ arbitrarily within their perturbative limits. ${ }^{2}$ We show the allowed region of these two gauge couplings in Fig. 5. While we still have a large region within perturbative limits, we have chosen $g_{R}$ to be either equal to $g_{L}$ or smaller while keeping $g_{B L}$ also below the order of one for our benchmark analysis. For $g_{R}>g_{R}$, the Planck bound becomes even more stringent, as we found in the scan plot shown in the $g_{R}-M_{W_{R}}$ plane in Fig. 4. Thus, compared to $U(1)_{B-L}$ or other Abelian gauge models of Dirac neutrinos, the DLRM is much more constrained. For a comparison, we show the parameter space for the gauged $U(1)_{B-L}$ model with Dirac neutrinos in Fig. 6. This is a minimal gauged $B-L$ model, where there are three right-handed neutrinos having $B-L$ charge -1 each apart from the SM fermion content and neutrinos get a sub-eV Dirac mass by virtue of their tiny couplings with the SM Higgs boson. Since the $B-L$ gauge coupling is a free parameter and not related to SM gauge couplings in this model, one can tune the gauge coupling arbitrarily to evade the stringent Planck bound on $\Delta N_{\text {eff }}$ as can be seen from Fig. 6 .

To check the impact of these constraints on DM parameter space, we then calculate the relic of right fermion quintuplet DM. For DM relic calculation, we first implement the model in SARAH [74] and then feed the model files into micrOMEGAs [75] for relic calculations. We then consider three benchmark combinations of $\left(g_{R}, W_{R}\right)$ while

\footnotetext{
${ }^{2}$ For a discussion related to perturbativity constraints on similar models, please refer to Ref. [73] and references therein.
}

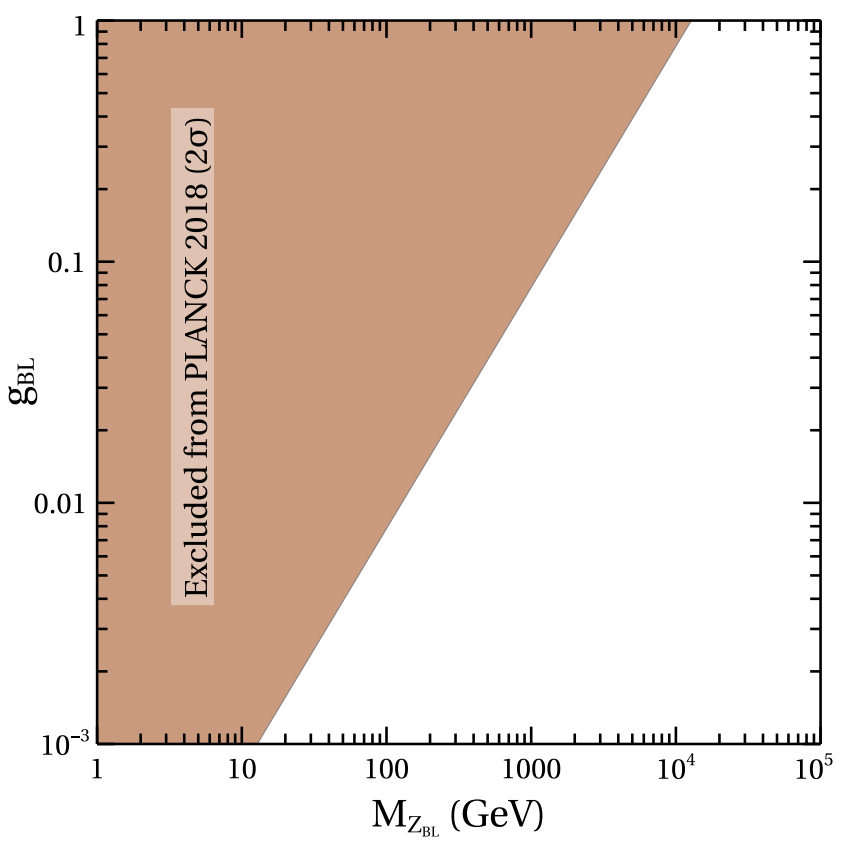

FIG. 6. Allowed $g_{B L}-M_{Z_{B L}}$ parameter space from the Planck 2018 bound on $\Delta N_{\text {eff }}$ at $2 \sigma$ in the minimal $U(1)_{B-L}$ gauge model with light Dirac neutrinos.

keeping the scale of left-right gauge symmetry breaking $v_{R}$ fixed. The resulting variation of DM relic as a function of the DM mass is shown in left panel in Fig. 7. The resonances corresponding to $W_{R}$ and $Z_{R}$ masses are clearly visible in this plot. Unlike in the quintuplet DM scenario in the triplet LRSM [58], here the two resonances are quite close to each other due to a smaller ratio of $Z_{R}$ to $W_{R}$ mass in the DLRM. The results shown in left panel in Fig. 7 also agree with that shown in Ref. [66]. We then scan the parameter space of $W_{R}$ and $\Omega_{R}^{0}$ masses and show the region satisfying the correct DM relic in the right panel in Fig. 7. Multiple allowed values of DM mass for a fixed $W_{R}$ mass are arising due to annihilation and coannihilations of $\Omega_{R}^{0}$, $\Omega_{R}^{ \pm}$, and $\Omega_{R}^{ \pm \pm}$mediated by $W_{R}$ and $Z_{R}$ bosons where $Z_{R}$ is slightly heavier than $W_{R}\left(M_{Z_{R}} \approx 1.2 M_{W_{R}}\right.$ in pure left-right symmetric limit $g_{R}=g_{L}$ ). Furthermore, the red and green solid lines in the right panel in Fig. 7 show the possibility of having three different values of DM mass with the correct relic abundance for a fixed $W_{R}$ mass. The same behavior is also seen in the left panel in Fig. 7 with a fixed $W_{R}$ mass, where the red and green lines satisfy the relic at three different values of DM mass. On the other hand, the blue dashed line in the left panel in Fig. 7 satisfies the correct relic abundance for five different values of DM mass, a feature which is also depicted by the blue solid line in the scan plot (right panel in Fig. 7). We also apply the corresponding bounds on the $W_{R}$ mass from Planck constraints on $\Delta N_{\text {eff }}$ at $2 \sigma$ C.L. as horizontal shaded lines so that the region below the respective lines is disallowed. Clearly, some part of the DM parameter space on the right 

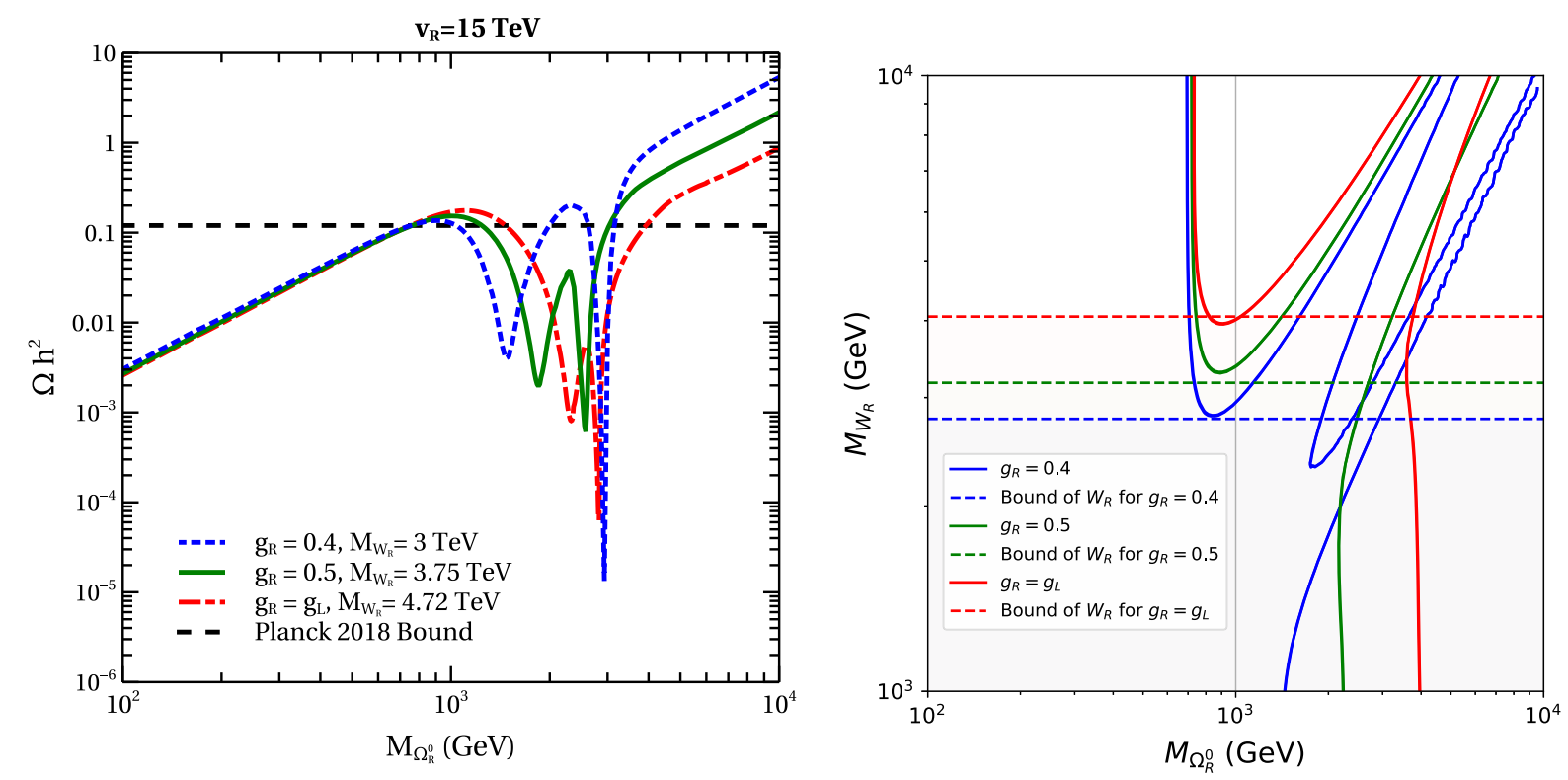

FIG. 7. Left panel: DM relic as a function of the DM mass for different benchmark combinations of $g_{R}$ and $W_{R}$. Right panel: Parameter space satisfying relic abundance criteria of right-handed fermion quintuplet dark matter in the DLRM. The shaded regions are ruled out by the Planck 2018 bound on $\Delta N_{\text {eff }}$ at $2 \sigma$ for respective values of $g_{R}$.

side of the scan plot (right panel in Fig. 7) gets disfavored for all three values of $g_{R}$ by $\Delta N_{\text {eff }}$ bounds, as the lower region of the dashed lines is excluded from Planck constraints on $\Delta N_{\text {eff }}$ at $2 \sigma$ C.L. In the parabolic part of the parameter space in the scan plot, a small part of the parameter space for the $g_{R}=g_{L}$ case is disfavored by $\Delta N_{\text {eff }}$ bounds, while the parabolic lines corresponding to $g_{R} \neq g_{L}$ still remain allowed from respective $\Delta N_{\text {eff }}$. We do not show other existing bounds on the $W_{R}$ mass from flavor or LHC data, as they are either equally or less strong compared to the bounds derived here.

\section{CONCLUSION}

We have studied the minimal left-right symmetric model with Higgs doublets, known as the doublet left-right model, where the left-right symmetry is broken spontaneously by a Higgs doublet instead of Higgs triplets in the LRSM with type-I plus type-II seesaw for light neutrino masses. In the minimal DLRM, a light neutrino mass can be realized only through the Higgs bidoublet with tiny coupling to neutrinos leading to a sub-eV Dirac neutrino mass. Because of $S U(2)_{R} \times U(1)_{B-L}$ gauge interactions, the right-handed neutrinos can thermalize in the early Universe, thereby contributing to the effective relativistic degrees of freedom $N_{\text {eff }}$ which are tightly constrained by CMB measurements. We constrain the scale of left-right symmetry from the requirement of satisfying the Planck 2018 bound on $\Delta N_{\text {eff }}$ not only for pure left-right symmetric limit $g_{R}=g_{L}$ but also for other values of $g_{R}$. While $g_{R}$ and $g_{B L}$ cannot be chosen arbitrarily in the LRSM due to their relation with $U(1)_{Y}$ coupling of the SM, the parameter space gets tightly constrained. For $g_{R}=g_{L}$, the Planck 2018 bound at $2 \sigma$ C.L. can rule out $W_{R}$ mass all the way up to $4.06 \mathrm{TeV}$, which is as competitive as the existing collider bounds on $W_{R}$ from dijet resonance searches. For example, the ATLAS dijet resonance search rules out such an additional charged vector boson mass up to $4 \mathrm{TeV}$ [20], while a similar analysis by CMS rules out up to $3.6 \mathrm{TeV}$ for SM-like gauge couplings [21]. Our conclusions also agree with the recent model-independent calculations [76] where, considering four fermion interactions of right-handed neutrinos and their contribution to $N_{\text {eff }}$, the authors constrained the interaction strength to be $10^{-5}-10^{-3}$ times the usual Fermi coupling constant. This bound we derive here, that is, $M_{W_{R}}>4.06 \mathrm{TeV}$, can be made weaker for smaller values of $g_{R}$. For example, in the case of $g_{R}=0.5$, we get $M_{W_{R}}>3.17 \mathrm{TeV}$ and $g_{R}=0.4$ leads to $M_{W_{R}}>2.77 \mathrm{TeV}$. We also make a comparison with similar constraints derived in the $U(1)_{B-L}$ gauge model with light Dirac neutrinos, where, due to the freedom in choosing the gauge coupling arbitrarily, one can have a much lighter $B-L$ gauge boson as well. However, the choices of $g_{R}$ as well as $g_{B L}$ are not arbitrary in the DLRM due to their nontrivial connection to $g_{Y}$ of the standard model. While we perform our analysis without explicitly solving the Boltzmann equations, the conclusions do not change much as long as the decoupling temperature of right-handed neutrinos remains much higher compared to that of active neutrinos. This was also noted by the authors of Ref. [76], and our parameter space is precisely confined to this regime.

We also show the impact of $\Delta N_{\text {eff }}$ constraints on dark matter parameter space in the DLRM. While the DLRM does not have a dark matter candidate on its own, we 

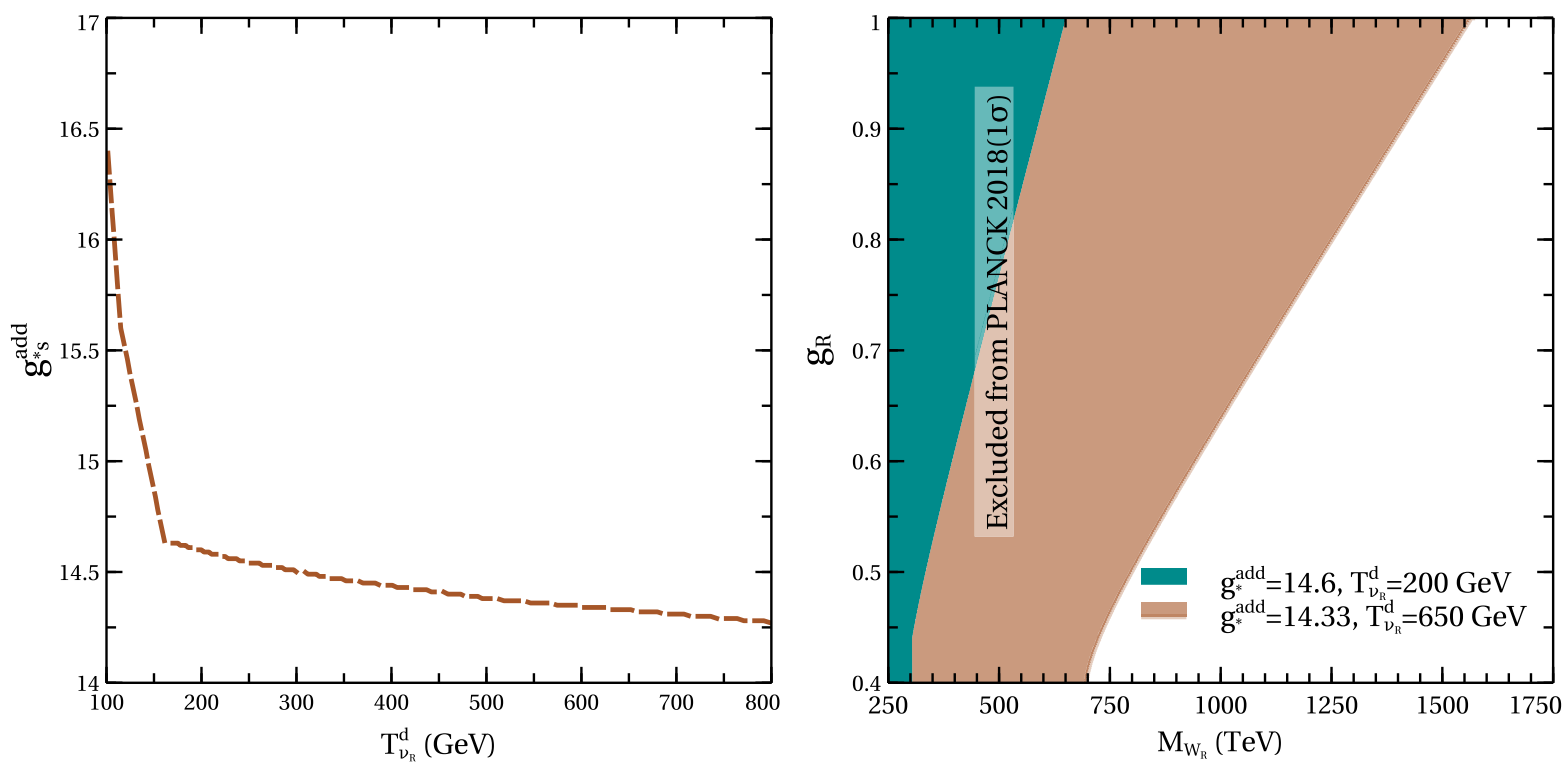

FIG. 8. Left panel: Additional relativistic degrees of freedom required to bring $N_{\text {eff }}$ within Planck $20181 \sigma$ bound $N_{\text {eff }}=2.99 \pm 0.17$. Right panel: Allowed parameter space in the $g_{R}-M_{W_{R}}$ plane from Planck $20181 \sigma$ bound $N_{\text {eff }}=2.99 \pm 0.17$ after considering additional relativistic degrees of freedom.

incorporate the presence of an additional fermion quintuplet DM in the minimal DM spirit. Since such a real fermion quintuplet does not have any renormalizable coupling with other fermions or scalars of the DLRM, the relic abundance of DM, the neutral component of the right-handed fermion quintuplet, depends crucially on its annihilation and coannihilation mediated by $W_{R}$ and $Z_{R}$ gauge bosons. We constrain the parameter space satisfying the correct DM relic by using the respective $\Delta N_{\text {eff }}$ bounds for different $g_{R}$. We find available parameter space satisfying the correct DM relic even after applying the Planck 2018 bound on $\Delta N_{\text {eff }}$ at $2 \sigma$ C.L.

We also compare our results in view of the more stringent Planck $20181 \sigma$ bound $N_{\text {eff }}=2.99 \pm 0.17$, which rules out all the parameter space if we assume only the SM plus three right-handed neutrinos to be contributing to the relativistic degrees of freedom (DOF) below the scale of left-right symmetry breaking. However, if there are more relativistic degrees of freedom due to the presence of light physical fields resulting from the scalar and fermion multiplets, one can satisfy the Planck $20181 \sigma$ bound as well. We show the required additional DOF in the left panel in Fig. 8 as a function of the right-handed neutrino decoupling temperature. All the points on the dashed line in the left panel in Fig. 8 give rise to $\Delta N_{\text {eff }}=0.12$, so that the points below this line are ruled out. These DOF can arise from the DLRM with right-handed fermion quintuplet DM. For example, the scalar bidoublet has four physical DOF apart from the SM Higgs boson, while the pair of Higgs doublets can give rise to five more physical DOF. Similarly, right-handed fermion quintuplet DM has approximately ten DOF. Thus, one can have 19 additional DOF in the DLRM with right-handed fermion quintuplet DM. Similar ways of avoiding such strict cosmological bounds on $\Delta N_{\text {eff }}$ have also been discussed in the recent work [76]. In the right panel in Fig. 8, we show the allowed parameter space on the $g_{R}-M_{W_{R}}$ plane from the Planck $20181 \sigma$ bound after considering the required additional relativistic DOF at decoupling temperatures of 200 and $650 \mathrm{GeV}$, respectively. Since a decoupling temperature of $200 \mathrm{GeV}$ can be achieved with a lighter $W_{R}$ gauge boson, we get more allowed parameter space compared to the case with a higher decoupling temperature of right-handed neutrinos. However, the presence of such additional light degrees of freedom will face stringent tests from collider as well as flavor physics constraints. Additionally, possible UV completion of this minimal model in order to explain the tiny origin of Dirac neutrino mass naturally [32-39] may involve additional interaction portals of right-handed neutrinos to thermalize with the standard bath. While the strength of these portals can be tuned with more freedom compared to the gauge portals discussed in this work and, hence, are unlikely to put stronger constraints than what we obtain here, it will be interesting to study the details of such scenarios, especially in the context of dark matter and collider phenomenology. A more detailed investigation of such scenarios is left for future studies.

\section{ACKNOWLEDGMENTS}

D. B. acknowledges support from Early Career Research Award from DST-SERB, Government of India (Reference No. ECR/2017/001873). C. M. thanks Supriya Senapati for useful discussion. 


\section{APPENDIX A: PHYSICAL MASSES OF GAUGE BOSONS}

Covariant derivatives of the scalar fields in the DLRM can be written as

$$
\begin{aligned}
D_{\mu} \Phi & =\partial_{\mu} \Phi-i \frac{g_{L}}{2}\left(\sigma \cdot W_{L \mu}\right) \Phi+i \frac{g_{R}}{2} \Phi\left(\sigma \cdot W_{R \mu}\right), \\
D_{\mu} \chi_{L} & =\partial_{\mu} \chi_{L}-i \frac{g_{L}}{2}\left(\sigma . W_{L \mu}\right) \chi_{L}-i g_{B L} Q_{B L} B_{\mu}^{\prime} \chi_{L}, \\
D_{\mu} \chi_{R} & =\partial_{\mu} \chi_{R}-i \frac{g_{R}}{2}\left(\sigma . W_{R \mu}\right) \chi_{R}-i g_{B L} Q_{B L} B_{\mu}^{\prime} \chi_{R},
\end{aligned}
$$

where

$$
\sigma . W_{L / R \mu}=\left(\begin{array}{cc}
W_{L / R \mu}^{3} & \sqrt{2} W_{L / R \mu}^{+} \\
\sqrt{2} W_{L / R \mu}^{-} & -W_{L / R \mu}^{3}
\end{array}\right)
$$

The corresponding kinetic Lagrangian of scalar fields is

$$
\begin{aligned}
\mathcal{L}_{\text {scalar }}= & \operatorname{Tr}\left[\left(D_{\mu} \Phi\right)^{\dagger}\left(D_{\mu} \Phi\right)\right]+\left(D_{\mu} \chi_{L}\right)^{\dagger}\left(D_{\mu} \chi_{L}\right) \\
& +\left(D_{\mu} \chi_{R}\right)^{\dagger}\left(D_{\mu} \chi_{R}\right) .
\end{aligned}
$$

Considering the scalar VEVs as

$$
\langle\Phi\rangle=\left(\begin{array}{cc}
\frac{k_{1}}{\sqrt{2}} & 0 \\
0 & \frac{k_{2}}{\sqrt{2}}
\end{array}\right), \quad\left\langle\chi_{L}\right\rangle=\left(\begin{array}{c}
0 \\
\frac{v_{L}}{\sqrt{2}}
\end{array}\right), \quad\left\langle\chi_{R}\right\rangle=\left(\begin{array}{c}
0 \\
\frac{v_{R}}{\sqrt{2}},
\end{array}\right)
$$

the charged vector boson mass matrix can be written as

$$
M_{V^{ \pm}}^{2}=\left(\begin{array}{cc}
\frac{1}{4} v_{L}^{2} g_{L}^{2}+\frac{1}{4}\left(k_{1}^{2}+k_{2}^{2}\right) g_{L}^{2} & -\frac{1}{2} g_{L} g_{R} k_{1} k_{2} \\
-\frac{1}{2} g_{L} g_{R} k_{1} k_{2} & \frac{1}{4} v_{R}^{2} g_{R}^{2}+\frac{1}{4}\left(k_{1}^{2}+k_{2}^{2}\right) g_{R}^{2}
\end{array}\right),
$$

whereas the neutral vector boson mass matrix is

$$
M_{V^{0}}^{2}=\left(\begin{array}{ccc}
\frac{1}{8} g_{L}^{2}\left(k_{1}^{2}+k_{2}^{2}+v_{L}^{2}\right) & -\frac{1}{4} g_{L} g_{R}\left(k_{1}^{2}+k_{2}^{2}\right) & -\frac{1}{4} g_{B L} g_{L} v_{L}^{2} \\
-\frac{1}{4} g_{L} g_{R}\left(k_{1}^{2}+k_{2}^{2}\right) & \frac{1}{8} g_{L}^{2}\left(k_{1}^{2}+k_{2}^{2}+v_{R}^{2}\right) & -\frac{1}{4} g_{B L} g_{R} v_{R}^{2} \\
-\frac{1}{4} g_{B L} g_{L} v_{L}^{2} & -\frac{1}{4} g_{B L} g_{R} v_{R}^{2} & \frac{1}{8} g_{B L}^{2}\left(v_{L}^{2}+v_{R}^{2}\right)
\end{array}\right) \text {. }
$$

As expected, the neutral gauge boson mass matrix has one vanishing eigenvalue, corresponding to the massless photon. After diagonalization of the mass matrices, we can represent the gauge fields in terms of physical gauge boson states as

$$
\begin{aligned}
W_{L_{\mu}}^{3} & =\frac{e}{g_{Y}} Z_{L \mu}+\frac{e}{g_{L}} A_{\mu}+0 Z_{R \mu}, \\
W_{R_{\mu}}^{3} & =-\frac{e g_{Y}}{g_{L} g_{R}} Z_{L \mu}+\frac{e}{g_{R}} A_{\mu}+\frac{g_{Y}}{g_{B L}} Z_{R \mu}, \\
B_{\mu}^{\prime} & =-\frac{e g_{Y}}{g_{L} g_{B L}} Z_{L \mu}+\frac{e}{g_{B L}} A_{\mu}-\frac{g_{Y}}{g_{R}} Z_{R \mu} .
\end{aligned}
$$

Also, we can express these couplings as $\sin \theta_{W}=\frac{e}{g_{L}}$ and $\cos \theta_{W}=\frac{e}{g_{Y}}$ with $\theta_{W}$ being the Weinberg angle. In the DLRM, $Z_{L \mu}$ and $Z_{R \mu}$ will also mix as the bidoublet $\Phi$ transforms nontrivially under both $S U(2)_{L}$ and $S U(2)_{R}$ gauge groups. The mixing can be represented as

$$
\begin{aligned}
& Z_{L \mu}=\cos \delta Z_{\mu}-\sin \delta Z_{\mu}^{\prime}, \\
& Z_{R \mu}=\sin \delta Z_{\mu}+\cos \delta Z_{\mu}^{\prime},
\end{aligned}
$$

where the mixing angle can be written as

$$
\tan 2 \delta=\frac{2\left(M_{L, R}^{0}\right)^{2}}{\left(M_{L}^{0}\right)^{2}-\left(M_{R}^{0}\right)^{2}}
$$

with

$$
\begin{gathered}
\left(M_{L}^{0}\right)^{2}=\frac{e^{2}\left(g_{L}^{2}+g_{Y}^{2}\right)^{2}\left(k_{1}^{2}+k_{2}^{2}+v_{L}^{2}\right)}{8 g_{L}^{2} g_{Y}^{2}}, \\
\left.\left(M_{L, R}^{0}\right)^{2}=\frac{e\left(g_{L}^{2}+g_{Y}^{2}\right)\left(g_{R}^{2}\left(k_{1}^{2}+k_{2}^{2}\right)-g_{B L}^{2} v_{L}^{2}\right)}{4 g_{B L} g_{L} g_{R}}, \quad \text { (A } 10\right) \\
\left(M_{R}^{0}\right)^{2}=\frac{g_{Y}^{2}\left(2 g_{B L}^{2} g_{R}^{2} v_{R}^{2}+g_{R}^{4}\left(k_{1}^{2}+k_{2}^{2}+v_{R}^{2}\right)+g_{B L}^{4}\left(v_{L}^{2}+v_{R}^{2}\right)\right)}{8 g_{B L}^{2} g_{R}^{2}}
\end{gathered}
$$

The charged vector boson states are

$$
\begin{aligned}
& W_{L \mu}^{ \pm}=\cos \zeta W_{1 \mu}^{ \pm}-\sin \zeta W_{2 \mu}^{ \pm}, \\
& W_{R \mu}^{ \pm}=\sin \zeta W_{1 \mu}^{ \pm}+\cos \zeta W_{2 \mu}^{ \pm}
\end{aligned}
$$

with

$$
\tan 2 \zeta=\frac{2 M_{L R}^{2}}{M_{L}^{2}-M_{R}^{2}}
$$

where

$$
M_{L}^{2}=\frac{1}{4} g_{L}^{2}\left(k_{1}^{2}+k_{2}^{2}+v_{L}^{2}\right),
$$




$$
\begin{gathered}
M_{R}^{2}=\frac{1}{4} g_{R}^{2}\left(k_{1}^{2}+k_{2}^{2}+v_{R}^{2}\right), \\
M_{L R}^{2}=-\frac{1}{2} g_{L} g_{R} k_{1} k_{2} .
\end{gathered}
$$

After diagonalization of $\left\{W_{L}^{ \pm}, W_{R}^{ \pm}\right\}$to $\left\{W_{1}^{ \pm}, W_{2}^{ \pm}\right\}$, we can have the corresponding mass-squared terms for the charged physical gauge bosons as

$$
\begin{aligned}
M_{1}^{2}= & \frac{1}{4}\left(g_{L}^{2}\left(k_{1}^{2}+k_{2}^{2}+v_{L}^{2}\right) \cos ^{2} \zeta-2 g_{L} g_{R} k_{1} k_{2} \sin 2 \zeta\right. \\
& \left.+g_{R}^{2}\left(k_{1}^{2}+k_{2}^{2}+v_{R}^{2}\right) \sin ^{2} \zeta\right), \\
M_{2}^{2}= & \frac{1}{4}\left(g_{R}^{2}\left(k_{1}^{2}+k_{2}^{2}+v_{R}^{2}\right) \cos ^{2} \zeta+2 g_{L} g_{R} k_{1} k_{2} \sin 2 \zeta\right. \\
& \left.+g_{L}^{2}\left(k_{1}^{2}+k_{2}^{2}+v_{L}^{2}\right) \sin ^{2} \zeta\right) .
\end{aligned}
$$

Note that we have taken $k_{2}=0$, which is equivalent to vanishing tree-level mixing angle $\zeta$. One can, however, generate radiative mixing between charged vector bosons, but that is typically very small $<10^{-7}$ [37]. Note that, although we write $W_{1}, W_{2}, Z$, and $Z^{\prime}$ as physical massive gauge boson states here to show the details, in the main text we continue to use $W_{L}, W_{R}, Z_{L}$, and $Z_{R}$ for better clarity.

\section{APPENDIX B: FERMION-GAUGE BOSON INTERACTIONS IN DLRM}

In this section, we note down the fermion interactions with massive vector bosons. The kinetic term of leptons in the DLRM is given by

$$
\mathcal{L}_{\ell}=i \overline{\ell_{L}} D^{L} \ell_{L}+i \overline{\ell_{R}} D^{R} \ell_{R}
$$

where
TABLE IV. Fermion interaction with the $Z^{\prime}$ boson.

$$
\begin{array}{lc}
\hline \hline \text { Fermions } & Z^{\prime} \text { in LRSM } \\
\overline{\overline{e_{L}}} \gamma_{\mu} e_{L} & i\left(\frac{g_{B L} g_{L} \tan \theta_{W} \cos \delta}{2 g_{R}}+\frac{g_{L} \cos 2 \theta_{W} \sin \delta}{\cos \theta_{W}}\right) \\
\overline{e_{R}} \gamma_{\mu} e_{R} & i\left(\frac{1}{2} g_{L} \tan \theta_{W}\left(\left(\frac{g_{B L}}{g_{R}}-\frac{g_{R}}{g_{B L}}\right) \cos \delta-2 \sin \theta_{W} \sin \delta\right)\right) \\
\overline{\nu_{L}} \gamma_{\mu} \nu_{L} & i\left(\frac{g_{B L} g_{L} \tan \theta_{W} \cos \delta}{2 g_{R}}-\frac{g_{L}\left(1-2 \sin ^{2} \theta_{W} \cos ^{2} \theta_{W}\right) \sin \delta}{2 \cos ^{2} \theta_{W} \sin \theta_{W}}\right) \\
\overline{\nu_{R}} \gamma_{\mu} \nu_{R} & i\left(\frac{\left.g_{B L}^{2}+g_{R}^{2}\right) g_{L} \tan \theta_{W} \cos \delta}{2 g_{B L} g_{R}}\right) \\
\overline{u_{L}} \gamma_{\mu} u_{L} & i\left(-\frac{g_{B L} g_{L} \tan \theta_{W} \cos \delta}{6 g_{R}}+g_{L} \sin \theta_{W}\left(-\frac{\cot \theta_{W}}{2}+\frac{\tan \theta_{W}}{6}\right) \sin \delta\right) \\
\overline{u_{R}} \gamma_{\mu} u_{R} & i\left(\frac{1}{6} g_{L} \tan \theta_{W}\left(-\frac{g_{B L}}{g_{R}}+\frac{3 g_{R}}{g_{B L}}\right) \cos \delta+4 \sin \theta_{W} \sin \delta\right) \\
\overline{d_{L}} \gamma_{\mu} d_{L} & i\left(-\frac{g_{B L} g_{L} \tan \theta_{W} \cos \delta}{6 g_{R}}+g_{L} \sin \theta_{W}\left(\frac{\cot \theta_{W}}{2}+\frac{\tan \theta_{W}}{6}\right) \sin \delta\right) \\
\overline{d_{R}} \gamma_{\mu} d_{R} & i\left(-g_{L} \tan \theta_{W}\left(\frac{\left.g_{B L}^{2}+3 g_{R}^{2}\right) \cos \delta}{6 g_{B L} g_{R}}+\frac{\sin \theta_{W} \sin \delta}{3}\right)\right) \\
\hline \hline & \partial_{\mu} \ell_{L}-i \frac{g_{L}}{2}\left(\sigma . W_{L \mu}\right) \ell_{L}+i \frac{g_{B L}}{2} B_{\mu} \ell_{L}, \\
D_{\mu}^{L} \ell_{L}= & (\mathrm{B} 2) \\
D_{\mu}^{R} \ell_{R}= & \partial_{\mu} \ell_{R}-i \frac{g_{R}}{2}\left(\sigma . W_{R \mu}\right) \ell_{R}+i \frac{g_{B L}}{2} B_{\mu} \ell_{R} .
\end{array}
$$

The same kinetic Lagrangian is, in fact, applicable to quarks, too, if we include gluons in the covariant derivative. We show the interactions with neutral massive vector bosons in Tables III and IV, respectively.

The interaction of neutrino $\nu$ and charged leptons $\ell$ with $W_{2}$ (or $W_{R}$ ) is similar to the ones with $W_{L}$ except that $g_{L}$ is replaced by $g_{R}$ :

$$
-\frac{i g_{R}}{\sqrt{2}} \gamma_{\mu} P_{R}
$$

where we have ignored the details of the right-handed lepton mixing matrix, taking it to be a unit matrix.

TABLE III. Fermion interactions with the $Z$ boson.

\begin{tabular}{lcc}
\hline \hline Fermions & $Z$ in SM & $Z$ bosons \\
\cline { 2 - 3 }$\overline{e_{L}} \gamma_{\mu} e_{L}$ & $-i \frac{g_{L}}{2 \cos \theta_{W}} \cos 2 \theta_{W}$ & in DLRM \\
$\overline{e_{R}} \gamma_{\mu} e_{R}$ & $i \frac{g_{L}}{\cos \theta_{W}} \sin ^{2} \theta_{W}$ & $i\left(-\frac{g_{L} \cos 2 \theta_{W}}{2 \cos \theta_{W}} \cos \delta+\frac{g_{B L} g_{L} \tan \theta_{W} \sin \delta}{2 g_{R}}\right)$ \\
$\overline{\nu_{L}} \gamma_{\mu} \nu_{L}$ & $i \frac{g_{L}}{2 \cos \theta_{W}}$ & $i\left(\frac{g_{L} \sin ^{2} \theta_{W} \cos \delta}{\cos \theta_{W}}+\frac{1}{2}\left(\frac{g_{B L}}{g_{R}}-\frac{g_{R}}{g_{B L}}\right) g_{L} \tan \theta_{W} \sin \delta\right)$ \\
$\overline{u_{L}} \gamma_{\mu} u_{L}$ & $-i \frac{g_{L}}{2 \cos \theta_{W}}\left(\cos ^{2} \theta_{W}-\frac{\sin ^{2} \theta_{W}}{3}\right)$ & $i\left(\frac{g_{L}\left(1-2 \sin ^{2} \theta_{W} \cos ^{2} \theta_{W}\right) \cos \delta}{2 \cos ^{2} \theta_{W} \sin \theta_{W}}+\frac{g_{B L} g_{L} \tan \theta_{W} \sin \delta}{2 g_{R}}\right)$ \\
$\overline{u_{R}} \gamma_{\mu} u_{R}$ & $-i \frac{2}{3} g_{L} \frac{\sin ^{2} \theta_{W}}{\cos \theta_{W}}$ & $i\left(-g_{L} \sin \theta_{W}\left(\frac{\cot \theta_{W}}{2}-\frac{\tan \theta_{W}}{6}\right) \cos \delta+\frac{g_{B L} g_{L} \tan \theta_{W} \sin \delta}{6 g_{R}}\right)$ \\
$\overline{d_{L}} \gamma_{\mu} d_{L}$ & $-i \frac{g_{L}}{2 \cos \theta_{W}}$ & $i\left(-\left(\frac{g_{L} \cos \theta_{W}}{2}+\frac{g_{L} \sin \theta_{W}}{6 \cos \theta_{W}}\right) \cos \delta-\frac{g_{B L} g_{L} \tan \theta_{W} \sin \delta}{6 g_{R}}\right)$ \\
$\left.\overline{d_{R}} \gamma_{\mu} d_{R}\left(-4 \sin \theta_{W} \cos \delta+\left(-\frac{g_{B L}}{g_{R}}+\frac{3 g_{R}}{g_{B L}}\right) \sin \delta\right)\right)$ \\
$\overline{\nu_{R}} \gamma_{\mu} \nu_{R}$ \\
\hline \hline
\end{tabular}




\section{APPENDIX C: ANNIHILATION CROSS SECTIONS OF RIGHT-HANDED NEUTRINOS}

The annihilation cross sections of $\nu_{R}$ mediated by right sector gauge bosons are

$$
\begin{gathered}
\sigma_{\nu_{R} \overline{\nu_{R}} \rightarrow q \bar{q}}=\frac{\left(a^{2}+b^{2}\right) \sqrt{1-\frac{4 m_{q}^{2}}{s}}\left(c^{2}\left(2 m_{q}^{2}+s\right)+d^{2}\left(s-4 m_{q}^{2}\right)\right)}{192 \pi M_{Z_{R}}^{4}}, \\
\sigma_{\nu_{R} \bar{\nu}_{R} \rightarrow \ell_{R} \bar{\ell}_{R}}=\frac{\sqrt{1-\frac{4 m_{\ell}^{2}}{s}}}{192 \pi M_{W_{R}}^{4} M_{Z_{R}}^{4}}\left(2 m_{\ell}^{2}\left(M_{W_{R}}^{4}\left(a^{2}+b^{2}\right)\left(c^{2}-2 d^{2}\right)-M_{W_{R}}^{2} M_{Z_{R}}^{2}(a+b)(c-2 d)-M_{Z_{R}}^{4}(a+b)\right)\right. \\
\left.+s\left(M_{W_{R}}^{4}\left(a^{2}+b^{2}\right)\left(c^{2}+d^{2}\right)-M_{W_{R}}^{2} M_{Z_{R}}^{2}(a+b)(c+d)+2 M_{Z_{R}}^{4}(a+b)\right)\right),
\end{gathered}
$$

where

$$
\begin{aligned}
& a=b=\frac{g_{L} \tan \theta_{W}}{2} \frac{g_{B L}^{2}+g_{R}^{2}}{2 g_{B L} g_{R}}, \\
& c= \begin{cases}\frac{g_{L} \tan \theta_{W}}{2} \frac{2 g_{B L}^{2}-g_{R}^{2}}{2 g_{B L} g_{R}} & \text { (for charged leptons), } \\
\frac{g_{L} \tan \theta_{W}}{2} \frac{3 g_{R}^{2}-2 g_{B L}^{2}}{6 g_{B L} g_{R}} & \text { (for up-type quark), } \\
-\frac{g_{L} \tan \theta_{W}}{2} \frac{2 g_{B L}^{2}+g_{R}^{2}}{2 g_{B L} g_{R}} & \text { (for down-type quark), }\end{cases} \\
& d= \begin{cases}-\frac{g_{L} \tan \theta_{W}}{4 g_{B L}} g_{R} & \text { (for charged leptons), } \\
\frac{g_{L} \tan \theta_{W}}{4 g_{B L}} g_{R} & \text { (for up-type quark), } \\
-\frac{g_{L} \tan \theta_{W}}{4 g_{B L}} g_{R} & \text { (for down-type quark). }\end{cases}
\end{aligned}
$$

We consider the mixing between left and right sector gauge bosons to be negligible and, hence, do not take it into account in our analysis.

[1] J. C. Pati and A. Salam, Lepton number as the fourth color, Phys. Rev. D 10, 275 (1974).

[2] R. N. Mohapatra and J. C. Pati, Left-right gauge symmetry and an isoconjugate model of $C P$ violation, Phys. Rev. D 11, 566 (1975).

[3] R. N. Mohapatra and J. C. Pati, A natural left-right symmetry, Phys. Rev. D 11, 2558 (1975).

[4] G. Senjanovic and R. N. Mohapatra, Exact left-right symmetry and spontaneous violation of parity, Phys. Rev. D 12, 1502 (1975).

[5] G. Senjanović, Spontaneous breakdown of parity in a class of gauge theories, Nucl. Phys. B153, 334 (1979).

[6] R. N. Mohapatra, F. E. Paige, and D. P. Sidhu, Symmetry breaking and naturalness of parity conservation in weak neutral currents in left-right symmetric gauge theories, Phys. Rev. D 17, 2462 (1978).

[7] R. N. Mohapatra and R. E. Marshak, Local B-L Symmetry of Electroweak Interactions, Majorana Neutrinos and Neutron Oscillations, Phys. Rev. Lett. 44, 1316 (1980).
[8] R. N. Mohapatra and G. Senjanovic, Neutrino masses and mixings in gauge models with spontaneous parity violation, Phys. Rev. D 23, 165 (1981).

[9] C. S. Lim and T. Inami, Lepton flavor nonconservation and the mass generation mechanism for neutrinos, Prog. Theor. Phys. 67, 1569 (1982).

[10] J. F. Gunion, J. Grifols, A. Mendez, B. Kayser, and F. I. Olness, Higgs bosons in left-right symmetric models, Phys. Rev. D 40, 1546 (1989).

[11] N. G. Deshpande, J. F. Gunion, B. Kayser, and F. I. Olness, Left-right symmetric electroweak models with triplet Higgs, Phys. Rev. D 44, 837 (1991).

[12] P. Fileviez Perez, Type III seesaw and left-right symmetry, J. High Energy Phys. 03 (2009) 142.

[13] R. N. Mohapatra et al., Theory of neutrinos: A white paper, Rep. Prog. Phys. 70, 1757 (2007).

[14] M. Tanabashi et al. (Particle Data Group Collaboration), Review of particle physics, Phys. Rev. D 98, 030001 (2018). 
[15] M. Aaboud et al. (ATLAS Collaboration), Search for a new heavy gauge boson resonance decaying into a lepton and missing transverse momentum in $36 \mathrm{fb}^{-1}$ of $p p$ collisions at $\sqrt{s}=13 \mathrm{TeV}$ with the ATLAS experiment, Eur. Phys. J. C 78, 401 (2018).

[16] M. Aaboud et al. (ATLAS Collaboration), Search for new phenomena in dijet events using $37 \mathrm{fb}^{-1}$ of $p p$ collision data collected at $\sqrt{s}=13 \mathrm{TeV}$ with the ATLAS detector, Phys. Rev. D 96, 052004 (2017).

[17] A. M. Sirunyan et al. (CMS Collaboration), Search for dijet resonances in proton-proton collisions at $\sqrt{s}=13 \mathrm{TeV}$ and constraints on dark matter and other models, Phys. Lett. B 769, 520 (2017).

[18] V. Khachatryan et al. (CMS Collaboration), Search for heavy gauge $\mathrm{W}^{\prime}$ boson in events with an energetic lepton and large missing transverse momentum at $\sqrt{s}=13 \mathrm{TeV}$, Phys. Lett. B 770, 278 (2017).

[19] A. M. Sirunyan et al. (CMS Collaboration), Search for highmass resonances in final states with a lepton and missing transverse momentum at $\sqrt{s}=13 \mathrm{TeV}$, J. High Energy Phys. 06 (2018) 128.

[20] G. Aad et al. (ATLAS Collaboration), Search for new resonances in mass distributions of jet pairs using $139 \mathrm{fb}^{-1}$ of $p p$ collisions at $\sqrt{s}=13 \mathrm{TeV}$ with the ATLAS detector, J. High Energy Phys. 03 (2020) 145.

[21] A. M. Sirunyan et al. (CMS Collaboration), Search for high mass dijet resonances with a new background prediction method in proton-proton collisions at $\sqrt{s}=13 \mathrm{TeV}$, J. High Energy Phys. 05 (2020) 033.

[22] V. Bernard, S. Descotes-Genon, and L. Vale Silva, Constraining the gauge and scalar sectors of the doublet leftright symmetric model, arXiv:2001.00886.

[23] P. Minkowski, $\mu \rightarrow e \gamma$ at a rate of one out of $10^{9}$ muon decays?, Phys. Lett. 67B, 421 (1977).

[24] M. Gell-Mann, P. Ramond, and R. Slansky, Complex spinors and unified theories, Conf. Proc. C 790927, 315 (1979).

[25] R. N. Mohapatra and G. Senjanovic, Neutrino Mass and Spontaneous Parity Violation, Phys. Rev. Lett. 44, 912 (1980).

[26] J. Schechter and J.W.F. Valle, Neutrino masses in $\mathrm{SU}(2) \times \mathrm{U}(1)$ theories, Phys. Rev. D 22, 2227 (1980).

[27] G. Lazarides, Q. Shafi, and C. Wetterich, Proton lifetime and fermion masses in an SO(10) model, Nucl. Phys. B181, 287 (1981).

[28] C. Wetterich, Neutrino masses and the scale of B-L violation, Nucl. Phys. B187, 343 (1981).

[29] J. Schechter and J. W. F. Valle, Neutrino decay and spontaneous violation of lepton number, Phys. Rev. D 25, 774 (1982).

[30] B. Brahmachari and R. N. Mohapatra, Unified explanation of the solar and atmospheric neutrino puzzles in a minimal supersymmetric SO(10) model, Phys. Rev. D 58, 015001 (1998).

[31] R. Foot, H. Lew, X. G. He, and G. C. Joshi, Seesaw neutrino masses induced by a triplet of leptons, Z. Phys. C 44, 441 (1989).

[32] K.S. Babu and X. G. He, Dirac neutrino masses as two loop radiative corrections, Mod. Phys. Lett. A 04, 61 (1989).
[33] E. Ma, Radiative Quark and Lepton Masses in a Left-right Gauge Model, Phys. Rev. Lett. 63, 1042 (1989).

[34] E. Ma and O. Popov, Pathways to naturally small Dirac neutrino masses, Phys. Lett. B 764, 142 (2017).

[35] D. Borah, Light sterile neutrino and dark matter in left-right symmetric models without a Higgs bidoublet, Phys. Rev. D 94, 075024 (2016).

[36] D. Borah and A. Dasgupta, Observable lepton number violation with predominantly Dirac nature of active neutrinos, J. High Energy Phys. 01 (2017) 072.

[37] D. Borah and A. Dasgupta, Naturally light Dirac neutrino in left-right symmetric model, J. Cosmol. Astropart. Phys. 06 (2017) 003.

[38] E. Ma and U. Sarkar, Radiative left-right Dirac neutrino mass, Phys. Lett. B 776, 54 (2018).

[39] H. Diaz Chavez, V. Pleitez, and O. Ravinez, Dirac neutrinos in a $S U(2)$ left-right symmetric model, arXiv:1908.02828.

[40] N. Aghanim et al. (Planck Collaboration), Planck 2018 results. VI. Cosmological parameters, arXiv:1807.06209.

[41] G. Mangano, G. Miele, S. Pastor, T. Pinto, O. Pisanti, and P. D. Serpico, Relic neutrino decoupling including flavor oscillations, Nucl. Phys. B729, 221 (2005).

[42] E. Grohs, G. M. Fuller, C. T. Kishimoto, M. W. Paris, and A. Vlasenko, Neutrino energy transport in weak decoupling and big bang nucleosynthesis, Phys. Rev. D 93, 083522 (2016).

[43] P. F. de Salas and S. Pastor, Relic neutrino decoupling with flavour oscillations revisited, J. Cosmol. Astropart. Phys. 07 (2016) 051.

[44] K. Abazajian et al., CMB-S4 science case, reference design, and project plan, arXiv:1907.04473.

[45] Y. Zhang, H. An, X. Ji, and R. N. Mohapatra, General CP violation in minimal left-right symmetric model and constraints on the right-handed scale, Nucl. Phys. B802, 247 (2008).

[46] K. N. Abazajian and J. Heeck, Observing Dirac neutrinos in the cosmic microwave background, Phys. Rev. D 100, 075027 (2019).

[47] P. Fileviez Pérez, C. Murgui, and A. D. Plascencia, Neutrino-dark matter connections in gauge theories, Phys. Rev. D 100, 035041 (2019).

[48] D. Nanda and D. Borah, Connecting light Dirac neutrinos to a multi-component dark matter scenario in gauged $B-L$ model, Eur. Phys. J. C 80, 557 (2020).

[49] C. Han, M. López-Ibáñez, B. Peng, and J. M. Yang, Dirac dark matter in $U(1)_{B-L}$ with Stueckelberg mechanism, arXiv:2001.04078.

[50] F. Zwicky, Die Rotverschiebung von extragalaktischen Nebeln, Helv. Phys. Acta 6, 110 (1933).

[51] V. C. Rubin and W. K. Ford, Jr., Rotation of the andromeda nebula from a spectroscopic survey of emission regions, Astrophys. J. 159, 379 (1970).

[52] D. Clowe, M. Bradac, A. H. Gonzalez, M. Markevitch, S. W. Randall, C. Jones, and D. Zaritsky, A direct empirical proof of the existence of dark matter, Astrophys. J. 648, L109 (2006).

[53] E. W. Kolb and M. S. Turner, The Early Universe (1990), Vol. 69.

[54] M. Cirelli, N. Fornengo, and A. Strumia, Minimal dark matter, Nucl. Phys. B753, 178 (2006). 
[55] C. Garcia-Cely, A. Ibarra, A. S. Lamperstorfer, and M. H. G. Tytgat, Gamma-rays from heavy minimal dark matter, J. Cosmol. Astropart. Phys. 10 (2015) 058.

[56] M. Cirelli, T. Hambye, P. Panci, F. Sala, and M. Taoso, Gamma ray tests of minimal dark matter, J. Cosmol. Astropart. Phys. 10 (2015) 026.

[57] J. Heeck and S. Patra, Minimal Left-Right Symmetric Dark Matter, Phys. Rev. Lett. 115, 121804 (2015).

[58] C. Garcia-Cely and J. Heeck, Phenomenology of left-right symmetric dark matter, J. Cosmol. Astropart. Phys. 03 (2016) 021.

[59] D. Borah, A. Dasgupta, and S. Patra, Common origin of $3.55 \mathrm{keV}$ x-ray line and gauge coupling unification with left-right dark matter, Phys. Rev. D 96, 115019 (2017).

[60] A. Berlin, P. J. Fox, D. Hooper, and G. Mohlabeng, Mixed dark matter in left-right symmetric models, J. Cosmol. Astropart. Phys. 06 (2016) 016.

[61] D. Borah, A. Dasgupta, U. K. Dey, S. Patra, and G. Tomar, Multi-component fermionic dark matter and IceCube PeV scale neutrinos in left-right model with gauge unification, J. High Energy Phys. 09 (2017) 005.

[62] P. S. B. Dev, D. Kazanas, R. N. Mohapatra, V. L. Teplitz, and Y. Zhang, Heavy right-handed neutrino dark matter and PeV neutrinos at IceCube, J. Cosmol. Astropart. Phys. 08 (2016) 034.

[63] P. S. Bhupal Dev, R. N. Mohapatra, and Y. Zhang, Naturally stable right-handed neutrino dark matter, J. High Energy Phys. 11 (2016) 077.

[64] P. S. B. Dev, R. N. Mohapatra, and Y. Zhang, Heavy righthanded neutrino dark matter in left-right models, Mod. Phys. Lett. A 32, 1740007 (2017).

[65] D. Borah and A. Dasgupta, Left right symmetric models with a mixture of keV-TeV dark matter, J. Phys. G 46, 105004 (2019).
[66] P. Ko and T. Nomura, $\mathrm{SU}(2)_{L} \times \mathrm{SU}(2)_{R}$ minimal dark matter with $2 \mathrm{TeV} W^{\prime}$, Phys. Lett. B 753, 612 (2016).

[67] D. Chang, R. N. Mohapatra, and M. K. Parida, Decoupling Parity and SU(2)-R Breaking Scales: A New Approach to Left-Right Symmetric Models, Phys. Rev. Lett. 52, 1072 (1984).

[68] D. Chang, R. N. Mohapatra, and M. K. Parida, A new approach to left-right symmetry breaking in unified gauge theories, Phys. Rev. D 30, 1052 (1984).

[69] D. Chang, R. N. Mohapatra, J. Gipson, R. E. Marshak, and M. K. Parida, Experimental tests of new $\mathrm{SO}(10)$ grand unification, Phys. Rev. D 31, 1718 (1985).

[70] K. Griest and D. Seckel, Three exceptions in the calculation of relic abundances, Phys. Rev. D 43, 3191 (1991).

[71] M. H. Abitbol et al. (Simons Observatory Collaboration), The Simons observatory: Astro2020 decadal project whitepaper, Bull. Am. Astron. Soc. 51, 147 (2019).

[72] B. Benson et al. (SPT-3G Collaboration), SPT-3G: A nextgeneration cosmic microwave background polarization experiment on the south pole telescope, Proc. SPIE Int. Soc. Opt. Eng. 9153, 91531P (2014).

[73] G. Chauhan, P. B. Dev, R. N. Mohapatra, and Y. Zhang, Perturbativity constraints on $U(1)_{B-L}$ and left-right models and implications for heavy gauge boson searches, J. High Energy Phys. 01 (2019) 208.

[74] F. Staub, SARAH 4: A tool for (not only SUSY) model builders, Comput. Phys. Commun. 185, 1773 (2014).

[75] G. Belanger, F. Boudjema, A. Pukhov, and A. Semenov, micrOMEGAs 3: A program for calculating dark matter observables, Comput. Phys. Commun. 185, 960 (2014).

[76] X. Luo, W. Rodejohann, and X.-J. Xu, Dirac neutrinos and $N_{\text {eff }}$, J. Cosmol. Astropart. Phys. 06 (2020) 058. 